\title{
Electroweak production of hybrid mesons in a Flux-Tube simulation of Lattice QCD
}

\author{
F.E.Close* and J.J.Dudeh \\ Department of Physics - Theoretical Physics, University of Oxford, \\ 1 Keble Rd., Oxford OX1 3NP, UK
}

\begin{abstract}
We make the first calculation of the electroweak couplings of hybrid mesons to conventional mesons appropriate to photoproduction and to the decays of $B$ or $D$ mesons. $E 1$ amplitudes are found to be large and may contribute in charge exchange $\gamma p \rightarrow n H^{+}$allowing production of (amongst others) the charged $1^{-+}$exotic hybrid off $a_{2}$ exchange. Axial hybrid meson photoproduction is predicted to be large courtesy of $\pi$ exchange, and its strange hybrid counterpart is predicted in $B \rightarrow \psi K_{H}\left(1^{+}\right)$ with b.r. $\sim 10^{-4}$. Higher multipoles, and some implications for hybrid charmonium are briefly discussed.
\end{abstract}

An outstanding problem in the Standard Model is how the non-Abelian, gluon, degrees of freedom behave in the limit of strong QCD. Lattice QCD predicts a spectroscopy of glueballs [1] and hybrid mesons [2], but there are no unambiguous signals against which these predictions can be tested.

A major stumbling block in the case of hybrids is that while predictions for their masses 2, 3], hadronic widths [4, 5] and decay channels [4, 5, 6] are rather well agreed upon, the literature contains no discussion of their production rates in electroweak interactions (beyond VMD in one exotic channel [7]). Meanwhile a significant plank in the proposed upgrade of Jefferson Laboratory is its assumed ability to expose the predicted hybrid mesons in photo and electroproduction. Also, high statistics studies of meson production in $B$ and $D$ decays are becoming available.

Clearly a calculation of hybrid production, appropriate to such experiments, in a model based on lattice QCD is urgently called for. Here we make the first direct calculation of electromagnetic and weak production of hybrids in such a model 3 . We find that the E1 transition amplitudes may be large and accessible in forthcoming experiments. Furthermore we predict that b.r. $\left[B \rightarrow \psi K_{H}\left(1^{+}\right)\right] \sim 10^{-4}$, and suggest that evidence for this may already be present in the enhancement of low momentum $\psi[\underline{8}]$.

\section{The model}

Theory 3, 9] has provided compelling arguments from QCD that confinement occurs via the formation of a flux tube: a relativistic object with an infinite number of degrees of freedom. A standard approximation [3, 4, 5, 10] has been to fix the longitudinal separation $\vec{r}=\vec{r}_{Q}-\vec{r}_{\bar{Q}}$ and to solve the flux-tube dynamics in the limit of a thin string with purely transverse degrees of freedom. The resulting energies $E(r)$ are then used as adiabatic effective potentials on which the meson spectroscopies are built. Ref. 11] studied the effect of relaxing these strict approximations and found that the spectrum of the conventional and lowest hybrids is robust. We shall assume the same is true in this first calculation of electroweak excitation of hybrid mesons.

In refs. 3, 10, 11] the flux-tube was discretised into $N+1$ cells, and then $N \rightarrow \infty$. Up to $N$ modes may be excited. We shall focus on the first excited state, with excitation energy $\omega=\pi / r$.

The flux tube is dynamic, with degrees of freedom in the two dimensions transverse to the $Q \bar{Q}$ axis. The state of the flux tube can be written in terms of a complete set of transverse eigenstates $\left|\vec{y}_{1} \ldots \vec{y}_{n} \ldots \vec{y}_{N}\right\rangle$ and the Fourier mode for the first excited state is

$$
\vec{y}_{n}=\sqrt{\frac{2}{N+1}} \vec{a}_{1} \sin \frac{\pi n}{N+1}
$$

In the small oscillation approximation the system becomes harmonic in $\vec{y}(\vec{a})$. The states of the flux-tube are then described by Gaussians (see eqs. $(11,12,13)$ in ref. 10]). For a pedagogic illustration, consider the tube to be modeled by a single bead, mass $b r$.

If the transverse displacement is $\vec{y}$, then conservation of the position of the centre of mass and of orbital angular momentum about the centre of mass leads to a mean transverse displacement of the $Q$ and $\bar{Q}$. If these have masses $m_{Q}$, then relative to the centre-of-mass, the position vector of the quark has components in the longitudinal $\vec{r}$ and transverse $\vec{y}$ directions

$$
\vec{r}_{Q}=\left[\frac{1}{2} \vec{r} ;\left(\frac{b r}{2 m_{Q}}\right) \vec{y}\right]
$$

The dependence of $\vec{r}_{Q}$ on $\vec{y}$ enables a quark-current interaction at $r_{Q}$ to excite transitions in the $\vec{y}$ oscillator, leading to excitation of the flux-tube.

This is the essential physics behind the excitation of hybrid modes by current interactions with the quark or antiquark. Extending to $N$ beads leads to more mathematical detail, but the underlying principles are the same. The position vector of the quark becomes 10.

$$
\vec{r}_{Q ; \bar{Q}}=\vec{R} \pm \frac{1}{2} \vec{r}+\frac{b r}{\pi m_{Q}} \sqrt{\frac{2}{N+1}} \vec{a}_{1}
$$

with $\vec{R}$ the position of the $q \bar{q}$-tube system centre-of-mass. 
It has been argued that this dependence $\vec{r}_{Q}=f(\vec{r}, \vec{y})$ gives significant contributions to static properties of hadrons, such as charge radii, $\left\langle r^{2}\right\rangle_{\pi}$ and to the slope of the Isgur-Wise function $\rho\left(v \cdot v^{\prime}\right)$ [10]. Specifically for $Q \bar{Q}$

$$
r_{Q}^{2}=\frac{1}{4}\left[1+\frac{8 b}{\pi^{3} m_{Q}^{2}} \sum_{1}^{\infty}\left(1 / p^{3}\right)\right]\left\langle r^{2}\right\rangle
$$

where the $\sum_{1}^{\infty}\left(1 / p^{3}\right) \sim 1.2$ arises from the sum over all modes contributing to zero-point oscillations of the fluxtube.

Isgur 10] showed that these "transverse excursions" give huge $\sim 51 \%$ corrections in light quark systems where $m_{Q}=m_{d}$, and $\sim 13 \%$ corrections in heavy-light $Q \bar{q}$ systems. Furthermore the $\sum_{1}^{\infty}\left(1 / p^{3}\right)$ is $\sim 80 \%$ saturated by its $p=1$ term. Together, these suggest that the transition amplitudes to the lowest hybrids ( $p=1$ phonon modes) could be substantial. We shall now demonstrate that this can be so, at least for certain quantum numbers. The respective amplitudes for conventional E1 transitions and the hybrid excitation come from expanding the incoming plane wave to leading order in the momentum transfer, thereby enabling the linear terms in $\vec{q} \cdot \vec{r}_{Q}$ to break the orthogonality of initial and final wavefunctions and cause the transition.

By combining with the tensor decomposition of the current-quark interaction, we may calculate excitation amplitudes to hybrids, and compare with those for conventional mesons in various multipoles. We will give extensive details elsewhere [12]. In this first note, we illustrate the principle in electromagnetic interactions and in what promises to be a prominent heavy flavour decay channel.

A general feature of operators required to excite the lowest hybrid states (the first flux-tube mode) is the presence of the transverse position vector $\vec{y}$ to break the orthogonality between the lowest $Q \bar{Q}$ state and the " $\vec{y}$ excited" hybrid states. Hence in photoproduction one accesses $E 1$ or (orbitally excited) $M 1$ transitions in leading order. These are $\Delta S=0$, e.g. $0_{Q}^{-+} \rightarrow 1_{H}^{ \pm \pm}$or $1_{Q}^{--} \rightarrow(0,1,2)_{H}^{\mp \pm}$. (Note that states with the "wrong" charge conjugation will only be accessible for flavoured mesons, e.g. in $\gamma p \rightarrow H^{+} n$, and hence will have no analogue for $c \bar{c}$ and other $I=0$ states).

Transitions involving spin-flip, $\Delta S=1$, will need a $\vec{\sigma}$ spin operator as well as the above. Such terms arise as finite size corrections to the $\vec{\sigma} \cdot \vec{B}$ magnetic interaction and also in the spin-orbit interaction $\vec{\sigma} \cdot \overrightarrow{p_{Q}} \times \vec{E}$, in $J_{e m}$. These are normally non-leading effects at $O(v / c)^{2}$ in amplitude and hence much suppressed for heavy flavours. They are known to give non-negligible contributions to some light flavour transitions. However, unlike the leading $\Delta S=0$ terms, their effects are less well defined (e.g. binding effects and other relativistic corrections can play a role at this order 13, 14]). It is results for the $\Delta S=0 \mathrm{E} 1$ tran- sitions that are most reliable and on which we primarily focus in this first evaluation.

\section{E1 excitations}

The familiar $E 1$ amplitude between $Q_{1} \bar{Q}_{2}$ conventional states (e.g $\gamma \pi \leftrightarrow b_{1}$ ) is

$$
\mathcal{M}\left(\gamma \pi \leftrightarrow b_{1}\right)=\left(\frac{e_{1}}{m_{1}}-\frac{e_{2}}{m_{2}}\right){ }_{b}\langle r\rangle_{\pi}|\vec{q}| \frac{\mu}{\sqrt{3}}
$$

where ${ }_{b}\langle r\rangle_{\pi}$ is the radial wavefunction moment $\int_{0}^{\infty} r^{2} d r R_{b}(r) r R_{\pi}(r)$, and $\mu$ is the reduced mass of the $Q \bar{Q}$. In line with ref. 10] we use constituent masses which subsume contributions from the string.

The analogous amplitude for exciting the $\vec{y}$ oscillator between spin singlet states leads to $\tilde{\mathcal{M}} \equiv \mathcal{M}\left(\delta_{+}-\delta_{-}\right)$ where

$$
\mathcal{M}\left(\gamma \pi \rightarrow a_{1 H}\right)=\left(\frac{e_{1}}{m_{1}}+\frac{e_{2}}{m_{2}}\right)_{H}\langle r\rangle_{\pi}|\vec{q}| \sqrt{\frac{b}{3 \pi^{3}}} \delta_{m,+1}
$$

(where the factors $\delta_{+,-}$refer to the flux tube $p=1$ phonon polarisation transverse to the body vector $\vec{r}$, while the $\delta_{m, \pm 1}$ refers to the hybrid polarisation in the fixed axes $x, y, z[3])$. The transition $\gamma \pi \leftrightarrow a_{1 H}$ is seen to vanish when $m_{1} \equiv m_{2}$ and $e_{1}=-e_{2}$ in accord with the constraints of $C$ conjugation. The above formula can be immediately taken over to flavoured states where $m_{1} \neq m_{2}$.

The parity eigenstates in the flux tube are given in ref. [3] Following that reference we denote the number of positive or negative helicity phonon modes by $\left\{n_{+}, n_{-}\right\}$, which for our present purposes will be $\{1,0\}$ or $\{0,1\}$. Parity eigenstates \pm are then the linear superpositions $\frac{1}{\sqrt{2}}(|\{1,0\}\rangle \mp|\{0,1\}\rangle)$ such that for $\pi \gamma E 1$ transitions we have

$$
\langle P=-\mid \pi \gamma\rangle=0 ; \quad\langle P=+\mid \pi \gamma\rangle=\sqrt{2} \mathcal{M} ;
$$

This applies immediately to the excitation of the hybrid $a_{1 H}^{ \pm}$in $\gamma \pi^{ \pm} \rightarrow a_{1 H}^{ \pm}$where there is no spin flip between the spin singlet $\pi$ and $a_{1 H}$. In general we can write the radiative width $\Gamma(A \rightarrow B \gamma)$ as

$$
4 \frac{E_{B}}{m_{A}} \frac{|\vec{q}|}{\left(2 J_{A}+1\right)} \sum_{m_{J}^{A}}\left|\sqrt{2} \mathcal{M}\left(m_{J}^{A}, m_{J}^{B}=m_{J}^{A}+1\right)\right|^{2}
$$

where the sum is over all possible helicities of the initial meson. The ratio of widths $\frac{\Gamma_{E 1}\left(a_{1 H}^{+} \rightarrow \pi^{+} \gamma\right)}{\Gamma_{E 1}\left(b_{1}^{+} \rightarrow \pi^{+} \gamma\right)}$ is then

$$
\frac{72}{\pi^{3}} \frac{b}{m_{n}^{2}}\left|\frac{{ }_{H}\langle r\rangle_{\pi}}{{ }_{b}\langle r\rangle_{\pi}}\right|^{2}\left[\frac{\left|\vec{q}_{H}\right|^{3} \exp \left(-\left|\vec{q}_{H}\right|^{2} / 8 \bar{\beta}_{H}^{2}\right)}{\left|\vec{q}_{b}\right|^{3} \exp \left(-\left|\vec{q}_{b}\right|^{2} / 8 \bar{\beta}_{b}^{2}\right)}\right]
$$


where the factor in square brackets includes the $q^{3}$ phasespace and a "typical" form-factor taken from the case of harmonic-oscillator binding 15$]$.

Compare the form of this ratio driven by eqs. (2) 3) with the transverse contribution to the elastic charge radius, eq.(1). In the approximation used here, the E1 transitions to the leading states saturate the dipole sum rule. This suggests the possibility of generalising some of our specific results into sum rules relating the elastic properties of hadrons to the excitation of their hybrid states [10].

In the Isgur-Paton adiabatic model 3$]$ with a variational harmonic-oscillator solution we obtain $\left|{ }_{H}\langle r\rangle_{\pi} /{ }_{b}\langle r\rangle_{\pi}\right|^{2} \approx$ 1.0 , so the radial moments do not suppress hybrids 12 . We follow ref. 3] and use the standard parameters $b=$ $0.18 \mathrm{GeV}^{2}, m_{n}=0.33 \mathrm{GeV}$ so that the prefactor $\frac{72}{\pi^{3}} \frac{b}{m_{n}^{2}} \approx$ 3.8 and hence there is no hybrid suppression from the flux-tube dynamics.

Within our variational solution $\beta_{H}=255 \mathrm{MeV}, \beta_{b}=$ $281 \mathrm{MeV}, \beta_{\pi}=335 \mathrm{MeV}$, so we see the $p=1$ hybrid state being of roughly the same size as the $L=1$ conventional state. The main uncertainty is the computed size of the $\pi$ 15. Assuming that this hybrid has mass $\sim 1.9 \mathrm{GeV}[2,3,11$, and using the measured width $\Gamma\left(b_{1}^{+} \rightarrow \pi^{+} \gamma\right)=230 \pm 60 \mathrm{keV}[16]$ we predict that

$$
\Gamma\left(a_{1 H}^{+} \rightarrow \pi^{+} \gamma\right)=2.1 \pm 0.9 \mathrm{MeV}
$$

where the error allows for the uncertainty in $\beta_{\pi}[12,15$, 17 .

The equivalent $E 1$ process for spin triplet $Q \bar{Q}$ states is $(0,1,2)_{H}^{+-} \leftrightarrow \rho \gamma$, where the only difference from the $S=0$ case is the addition of $L, S$ Clebsch-Gordan factors coupling the $Q \bar{Q}$ spin and flux-tube angular momentum to the total $J$ of the hybrid meson in question. The matrix element is analogous to eq.(3) multiplied by the Clebsch-Gordan $\left\langle 1+1 ; 1 m_{\rho} \mid J m_{J}\right\rangle$. We find (for $J=0,1,2$ in this $E 1$ limit),

$$
\Gamma\left(b_{J H}^{+} \rightarrow \rho^{+} \gamma\right)=2.3 \pm 0.8 \mathrm{MeV} .
$$

where the error reflects the uncertainties in the conventional $E 1$ strength and $\beta_{f_{1}}$ and where we have taken $m_{H}=1.9 \mathrm{GeV}$.

\section{Heavy Flavor Decays}

As discussed after eq.(11), the $|\mathcal{M}|^{2}$ for the weak transition $B \rightarrow \psi K_{H}\left(1^{+}\right)$is expected to have strength $\sim 13 \%$ relative to its "conventional" counterpart $B \rightarrow \psi K\left(1^{+}\right)$. Empirically $B^{+} \rightarrow \psi K\left(1^{+}\right)(1280)$ is the single largest branching mode in $B^{+} \rightarrow \psi X$ with b.r. $=(1.8 \pm 0.5) \times$ $10^{-3}$ while $B^{+} \rightarrow \psi K\left(1^{+}\right)(1400) \leq 0.5 \times 10^{-3}$. These rates involve both parity conserving (vector) and violating (axial) contributions and their relative strengths depend on the mixing between the ${ }^{3} P_{1}$ and ${ }^{1} P_{1}$ basis states.

\begin{tabular}{c|c|c|c} 
state & & $u \bar{d}$ & $u \bar{s}$ \\
\hline${ }^{1} S_{0}$ & $\times 1$ & $\gamma \pi^{+} \rightarrow a_{1 H}^{+}$ & $\gamma K^{+} \rightarrow K_{1 A H}^{+}$ \\
& $56(23)$ & $43(23)$ \\
${ }^{3} S_{1}$ & $\times\left\langle 11 ; 1 m_{i} \mid J_{H} m_{H}\right\rangle$ & $\gamma \rho^{+} \rightarrow b_{J H}^{+}$ & $\gamma K^{*+} \rightarrow K_{J B H}^{+}$ \\
& 56 & 43 \\
${ }^{1} P_{1}$ & $\times \sqrt{\frac{3}{2}}\left\langle 11 ; 1 m_{i} \mid 1 m_{H}\right\rangle$ & $\gamma b_{1}^{+} \rightarrow \rho_{H}^{+}$ & $\gamma K_{1 B}^{+} \rightarrow K_{H}^{*+}$ \\
& $\times \sum^{3} P_{J}$ & 87 & 68 \\
& $\times \sum_{m_{L}, m_{S}}\left\langle 1 m_{L} ; 1 m_{S} \mid J m_{J}\right\rangle$ & $\gamma a_{J}^{+} \rightarrow \pi_{J_{H} H}^{+}$ & $\gamma K_{J A}^{+} \rightarrow K_{J_{H} H}^{+}$ \\
& $\sqrt{\frac{3}{4}}\left\langle 1 m_{L}+1 ; 1 m_{S} \mid J_{H} m_{H}\right\rangle^{*}$ & 87 & 68 \\
\hline
\end{tabular}

TABLE I: Photon-Meson-Hybrid matrix elements: $\sqrt{2} \mathcal{M}=$ $\left(\frac{e_{1}}{m_{1}}+\frac{e_{2}}{m_{2}}\right) \sqrt{2}|\vec{q}| \sqrt{\frac{b}{3 \pi^{3}}} H\langle r\rangle_{i}$ should be multiplied by the Clebsch-Gordan factor in the second column to give the overall matrix element for a positive helicity photon. The numbers quoted in columns three and four are $\sqrt{2} \mathcal{M} /|\vec{q}|\left(10^{-3} \mathrm{GeV}^{-1}\right)$, evaluated using the results of [3], except those in brackets which use the $\beta$-values of [17].

These rates would lead one to expect an order of magnitude b.r. for $B^{+} \rightarrow \psi K_{H}\left(1^{+}\right) \geq 10^{-4}$.

Explicit calculation confirms this. (For technical reasons our analysis of heavy-light dynamics is not identical to the original formulation of [10]. Details are in [18]). The transition matrix element has the structure

$$
\mathcal{M} \sim\left\langle K_{H}\left|V_{\mu}-A_{\mu}\right| B\right\rangle f_{\psi} m_{\psi} \epsilon_{\psi}^{* \mu}
$$

where $f_{\psi}=0.4 \mathrm{GeV}[19]$. A non-relativistic expansion of the vector and axial operators is made for both longitudinal and transverse components and terms linear in $\vec{y}$ or $\vec{p}_{y}$ identified. This is algebraically tedious but in essence parallels the approach illustrated earlier. The expectation values of these linear terms in $\vec{y}$ space generate the transitions to hybrid $K_{H}\left(1^{+}\right)$; the analogous terms in $\vec{r}$ space lead to the familiar $K\left(1^{+}\right)$states.

For $\Delta S=0$ transitions $B \rightarrow \psi K_{H}\left(1^{ \pm}\right)$,

$$
V_{\mu} \sim p_{\vec{y}}^{\mu} \frac{m_{b}+m_{s}}{m_{b} m_{s}} ; A_{\mu} \rightarrow A_{T} \sim|\vec{q}| p_{\vec{y}}^{(T)} / m_{b} m_{s}
$$

Hence the transition to $K_{H}\left(1^{+}\right)$is large because the dominant $\left\langle V_{\mu}\right\rangle$ contributes in S-wave; by contrast $K_{H}\left(1^{-}\right)$receives its S-wave from the $|\vec{q}| / m_{b}$-suppressed $\left\langle A_{\mu}\right\rangle$ while the vector current contributes to $\mathrm{P}$-waves. Explicit calculation confirms this where as a function of $m_{H}=$ $(1.8 ; 2.0 ; 2.2) \mathrm{GeV}$ we find

$$
\begin{aligned}
& \operatorname{b.r}\left[B \rightarrow \psi K_{H}\left(1^{-}\right)\right]=(1.2 ; 0.5 ; 0.2) \times 10^{-5} \\
& b . r\left[B \rightarrow \psi K_{H}\left(1^{+}\right)\right]=(4.5 ; 2.3 ; 1.0) \times 10^{-4} .
\end{aligned}
$$

Furthermore we find that $K_{H}\left(1^{+}\right)$is dominantly produced with longitudinal polarisation.

While fine details of the model may be questioned, the $O\left(10^{-4}\right)$ branching ratio to this hybrid appears robust 
and accessible to experiment. It is intriguing therefore that there is an unexplained enhancement at low $q_{\psi}$, corresponding to high mass $K$ systems, of this magnitude [8]. While suggestive, it would be premature to claim this as evidence for hybrid production. Radial excitations of the $K\left(1^{+}\right)$are expected in this region, and in the ISGW 20. model, extended to exclusive hadronic decays and assuming standard factorisation arguments 21], we find these to have b.r. $\sim 10^{-4}$, though slightly less than the hybrid. Other strange mesons in this mass range are likely to be suppressed due to their high angular momentum which give powerful orthogonality suppressions at small $q$. It is the S-wave character of the hybrid and axial production that drives their significant production rates.

To test these predictions experimentally, first identify the $\psi$ vertex and reconstruct the B from the decay hadrons and thereby the invariant mass distribution of the strange system. Observation of significant axial strength around $2 \mathrm{GeV}$, produced by parity conserving S-wave amplitudes at b.r. $>10^{-4}$ would prove strong evidence for the presence of the hybrid meson and warrant further studies of how to quantify the relative production and mixing of these axial mesons. In turn it would underpin our predictions of significant E1 transitions to such states in photoproduction.

There is also the possibility of hybrid Charmonium in $B \rightarrow \psi_{H} X$. Predicting this involves knowledge of flux-tube formation dynamics [22] which goes beyond the present work.

\section{Conclusions}

We confirm Isgur's conjecture that electromagnetic transitions to hybrids may be significant. We find this to be true for certain $E 1$ transitions for light flavours in charge exchange.

Within this model we also anticipate that $g g$ interactions initiate significant cascades such as $\psi_{H} \rightarrow \psi \eta(\eta \prime)$ and the diffractive transition $\gamma N \rightarrow 2_{H}^{+-} N$; these currents will disturb the flux tube by direct analogy with the electromagnetic transitions discussed here.

These results promise an active programme of future research at an upgraded Jefferson Laboratory and at CLEO-c. They also encourage mining existing data on $B$ decays and inclusion in future plans for heavy flavor decays. In particular there is the intriguing observation of an as yet unexplained enhancement in $B \rightarrow \psi X$ in the kinematic region where $K_{H}$ is expected, and with a strength compatible with that predicted for $K_{H}=1^{+}$. We urge further investigation of this, and the other channels identified in this note.

We shall give a detailed discussion elsewhere 12 .

\section{Acknowledgments}

This work is supported, in part, by grants from the Particle Physics and Astronomy Research Council, and the EU-TMR program "Euridice", HPRN-CT-2002-00311. We thank P.J.S. Watson for discussions on the dynamics of flux tubes.

* Electronic address: F.Close1@physics.ox.ac.uk

$\dagger$ Electronic address: dudek@thphys.ox.ac.uk

[1] SESAM Collaboration, G. Bali et al. Nucl.Phys.Proc.Suppl. 63, 209 (1997); IBM Collaboration, J. Sexton et al., Phys.Rev.Lett. 75, 4563 (1995); F.E. Close and M.J. Teper, Report no. RAL96-040/OUTP-96-35P; C.J. Morningstar and M. Peardon, Phys.Rev. D 56, 4043 (1997); D. Weingarten, Nucl.Phys.Proc.Suppl. 53, 232 (1997), ibid 63, 194 (1998), ibid 73, 249 (1999); C. McNeile and C. Michael, Phys. Rev. D 63, 114503, (2001)

[2] P. Lacock, C. Michael, P. Boyle and P. Rowland, Phys.Lett. B 401, 308 (1997); C. Bernard et al., Phys.Rev. D 56, 7039 (1997); P. Lacock and K. Schilling, Nucl. Phys. Proc. Suppl. 73, 261 (1999); C. McNeile, hep-lat/9904013 C. Morningstar, Nucl. Phys. Proc. Suppl. 90, 214 (2000)

[3] N. Isgur and J. Paton, Phys. Rev. D 31, 2910 (1985)

[4] N. Isgur, R. Kokoski and J. Paton, Phys. Rev. Lett. 54, 869 (1985)

[5] F.E. Close and P.R. Page, Nucl.Phys. B 443, 233 (1995)

[6] C. Michael, hep-ph/0009115

[7] A.Afanasev and P.R.Page, Phys.Rev.D 57, 6771 (1998)

[8] BABAR Collaboration, B.Aubert et al.,Phys.Rev. D67,032002 (2003)

[9] "Quark Confinement and the Hadron Spectrum II", ed. N. Brambilla and G.M. Prosperi (World Scientific, Singapore,1997)

[10] N. Isgur, Phys.Rev. D 60, 114016, (1999)

[11] T. Barnes, F.E. Close, and E.S. Swanson, Phys.Rev. D 52, 5242 (1995)

[12] F.E. Close and J.J. Dudek, "Hybrid meson production by electromagnetic and weak interactions in a Flux-Tube simulation of Lattice QCD" (in preparation)

[13] A. Le Yaouanc, L. Oliver, O. Pene and J.C. Raynal, Z.Phys. C 40, 77 (1988)

[14] F.E. Close and H. Osborn, Phys.Lett. B 34400 (1971); G. Hardekopf and J. Sucher, Phys.Rev. D 252938 (1982); R. McClary and N. Byers, Phys.Rev. D 281692 (1983)

[15] F.E. Close, A. Donnachie and Yu.S. Kalashnikova, Phys.Rev. D 65, 092003 (2002)

[16] Particle Data Group, Eur.Phys.J. C 15, 1 (2000)

[17] E.S. Swanson, Ann. Phys. 220, 73 (1992)

[18] J.Dudek, "The structure of $B$ and $D$ hybrid states in the flux tube model" (in preparation)

[19] M.Beneke, A.Signer and V.A.Smirnov, Phys.Rev.Lett. 80, 2535 (1998)

[20] N.Isgur, D.Scora, B.Grinstein and M.Wise, Phys.Rev. D39,799 (1989)

[21] M.Bauer, B.Stech, M.Wirbel, Z.Phys. C 34, 103 (1987); H.Y.Cheng, Y.Y.Keum and K.C.Yang, Phys.Rev.D 65, $094023(2002)$

[22] G.Chiladze, A.F.Falk and A.A.Petrov, Phys.Rev.D 58, 034013 (1998) 